\title{
Extending Causality Tests with Genetic Instruments: An Integration of Mendelian Randomization with the Classical Twin Design
}

\author{
Camelia C. Minică $^{1} \cdot$ Conor V. Dolan ${ }^{1} \cdot$ Dorret I. Boomsma $^{1} \cdot$ Eco de Geus $^{1} \cdot$ Michael C. Neale $^{1,2}$
}

Received: 2 September 2017 / Accepted: 24 May 2018 / Published online: 7 June 2018

(c) The Author(s) 2018

\begin{abstract}
Although experimental studies are regarded as the method of choice for determining causal influences, these are not always practical or ethical to answer vital questions in health and social research (e.g., one cannot assign individuals to a "childhood trauma condition" in studying the causal effects of childhood trauma on depression). Key to solving such questions are observational studies. Mendelian Randomization (MR) is an influential method to establish causality in observational studies. MR uses genetic variants to test causal relationships between exposures/risk factors and outcomes such as physical or mental health. Yet, individual genetic variants have small effects, and so, when used as instrumental variables, render MR liable to weak instrument bias. Polygenic scores have the advantage of larger effects, but may be characterized by horizontal pleiotropy, which violates a central assumption of MR. We developed the MR-DoC twin model by integrating MR with the Direction of Causation twin model. This model allows us to test pleiotropy directly. We considered the issue of parameter identification, and given identification, we conducted extensive power calculations. MR-DoC allows one to test causal hypotheses and to obtain unbiased estimates of the causal effect given pleiotropic instruments, while controlling for genetic and environmental influences common to the outcome and exposure. Furthermore, the approach allows one to employ strong instrumental variables in the form of polygenic scores, guarding against weak instrument bias, and increasing the power to detect causal effects of exposures on potential outcomes. Beside allowing to test pleiotropy directly, incorporating in MR data collected from relatives provide additional within-family data that resolve additional assumptions like random mating, the absence of the gene-environment interaction/covariance, no dyadic effects. Our approach will enhance and extend MR's range of applications, and increase the value of the large cohorts collected at twin/family registries as they correctly detect causation and estimate effect sizes even in the presence of pleiotropy.
\end{abstract}

Keywords Causality $\cdot$ Pleiotropy $\cdot$ Twin design $\cdot$ Mendelian randomization

\section{Introduction}

Edited by Sarah Medland.

Electronic supplementary material The online version of this article (https://doi.org/10.1007/s10519-018-9904-4) contains supplementary material, which is available to authorized users.

Camelia C. Minică

camelia.minica@vu.nl

1 Department of Biological Psychology, Vrije Universiteit Amsterdam, Transitorium 2B03, Van der Boechorststraat 1, 1081 BT Amsterdam, The Netherlands

2 Virginia Institute for Psychiatric and Behavioral Genetics, Virginia Commonwealth University, 1-156, P.O. Box 980126 , Richmond, VA 23298-0126, USA
Establishing causality in observational studies is important as knowledge of the relationship between a putative causal factor (exposure) and a potential outcome may inform rational treatment and prevention policies. While randomized controlled trials (RCTs) are the acid test of causality, they are expensive, time consuming, and may be practically or ethically unfeasible. For example, one cannot assign randomly individuals to a 'childhood trauma condition' in studying the causal effects of' childhood trauma' on'depression'. An important alternative to the RCT is Mendelian Randomization (Davey Smith and Ebrahim 2003).

Mendelian Randomization (MR) offers some traction in addressing causality by using genetic variants as instrumental variables to detect the causal effect of a modifiable risk 
factor (exposure) on a disease outcome in non-experimental settings (Evans and Davey Smith 2015; Davey-Smith and Hemani 2014). MR is quickly becoming one of the dominant approaches to establishing causality; many recent applications have been published (Evans and Davey Smith 2015; Ference et al. 2012, 2015; Vimaleswaran et al. 2013; Holmes et al. 2014a, b, c; Proitsi et al. 2014). The ascendency of MR is due to: dramatic drop in DNA genotyping costs, which has given rise to large samples of genotyped individuals, robust genetic associations established in genome-wide association studies (GWASs) (Welter et al. 2014), and the inherent advantages of MR, which include ecologic validity, robustness to reverse causation (from exposure to instrument) (Davey-Smith 2011) and confounding (Burgess and Thompson 2015).

MR requires instruments with a relatively strong association with the exposure. A disadvantage of many genetic variants is that they have weak effects (Visscher et al. 2012). Weak instruments confer insufficient statistical power, and render MR liable to weak instrument bias (Burgess and Thompson 2015; Davies et al. 2015; Burgess et al. 2011). Combining the weak genetic effects into a polygenic risk score (PGS) is a possible route to increase the strength of the genetic instrument (Burgess and Thompson 2013, 2015; Palmer et al. 2012; Pierce et al. 2010). However, the MR assumption that the instrument is not pleiotropic (has no direct effect on the outcome) is stronger in the case of a PGS instrument (Burgess and Thompson 2015; Burgess et al. 2011, 2014; Sheehan et al. 2008). A PGS comprises many genetic variants, any of which may directly affect both the exposure and the outcome, or may include variants in linkage disequilibrium with variants affecting the outcome. As demonstrated by twin studies (de Geus 2006; Kendler et al. 1992; Ligthart and Boomsma 2012; Middeldorp et al. 2005; Neale and Kendler 1995) and by polygenic risk score analyses (Evans et al. 2009; Purcell et al. 2009; Ligthart et al. 2014), many genetic variants associate with multiple phenotypes, suggesting pervasive pleiotropy (Sivakumaran et al. 2011; Bulik-Sullivan et al. 2015; Solovieff et al. 2013; Pickrell et al. 2016; Visscher and Yang 2016; Evans et al. 2013).

Several methods are currently in use as means to tackle the 'no pleiotropy' assumption [see also e.g., (Burgess et al. 2017; van Kippersluis and Rietveld 2017)]. Some approaches apply prior selection criteria to increase the probability that the instruments are valid. For instance, the stepwise procedure implemented in the R-package gtx (Johnson and Johnson 2012) employs iteratively a heterogeneity test to discard from a polygenic score genetic instruments yielding significant heterogeneity in the estimates of the causal effect. Possible heterogeneity is assumed to be indicative of pleiotropy. The efficiency of this method depends on its power to detect heterogeneity arising from pleiotropy. However, heterogeneity may arise from sources other than pleiotropy, so that one may needlessly weaken the instrument by removing valid genetic variants. Stepwise heterogeneity tests for identifying pleiotropy may suffer also from over-fitting, and may become difficult to interpret in the presence of many pleiotropic instruments. As acknowledged by the authors, the performance of this procedure in terms of bias and type I error rates within the MR context has yet to be established. Other approaches like those based on the median estimator (Bowden et al. 2016) can handle information from up to $50 \%$ invalid instruments. However, the strong MR assumption still applies to the variant(s) yielding the median causal effect. MR-Egger regression (Bowden et al. 2015) is another approach that, with weaker assumptions, gives consistent estimates even when all instruments are pleiotropic. The estimator is (asymptotically) consistent (i.e., the estimate of the causal effect converges to the true value with increasing sample size) under the assumption that the effect of the instrument on the exposure is uncorrelated with the direct effect of the instrument on the outcome (i.e., the Instrument Strength Independent of Direct Effect assumption). As the authors note, this assumption may not hold universally (Bowden et al. 2015). Furthermore, Bowden et al. noted (Bowden et al. 2015) that there are other plausible paths from the instrument to the outcome (in addition to the direct path, or the indirect path, via the exposure). For example, a possible path is via confounders affecting both traits, or due to linkage disequilibrium between the instrument and a genetic variant affecting the outcome. In this case the estimate of the estimates of the causal effect in Egger regression will likely be biased (Bowden and Jackson 2015). Finally, although MR-Egger uses multiple genetic variants to estimate the causal effect, these instruments are employed individually (i.e., not combined in a polygenic score). Strong instruments in the form of polygenic scores are desirable, not only to ensure sufficient statistical power, but also to preclude weak instrument bias.

The utility of the classical twin design (CTD) in the study of direction of causality is well established (De Moor et al. 2008; Duffy and Martin 1994; Heath et al. 1993; Kohler et al. 2011; Turkheimer and Harden 2000). The present aim is to combine MR and CTD into a single model. A similar approach was proposed by Kohler et al. who integrated CTD with the instrumental variable method (Kohler et al. 2011). We focus on the issues of identification and statistical power associated specifically with a (poly-)genic instrument, which may be related directly to the outcome (i.e., violating the 'no-pleiotropy' assumption). Integrating MR with CTD has three advantages: (1) it allows one to relax the strong MR assumption concerning the instrument's conditional independence of the outcome (conditional on the exposure and confounders, i.e., the no pleiotropy assumption, also known as the exclusion restriction assumption; (2) by accounting 


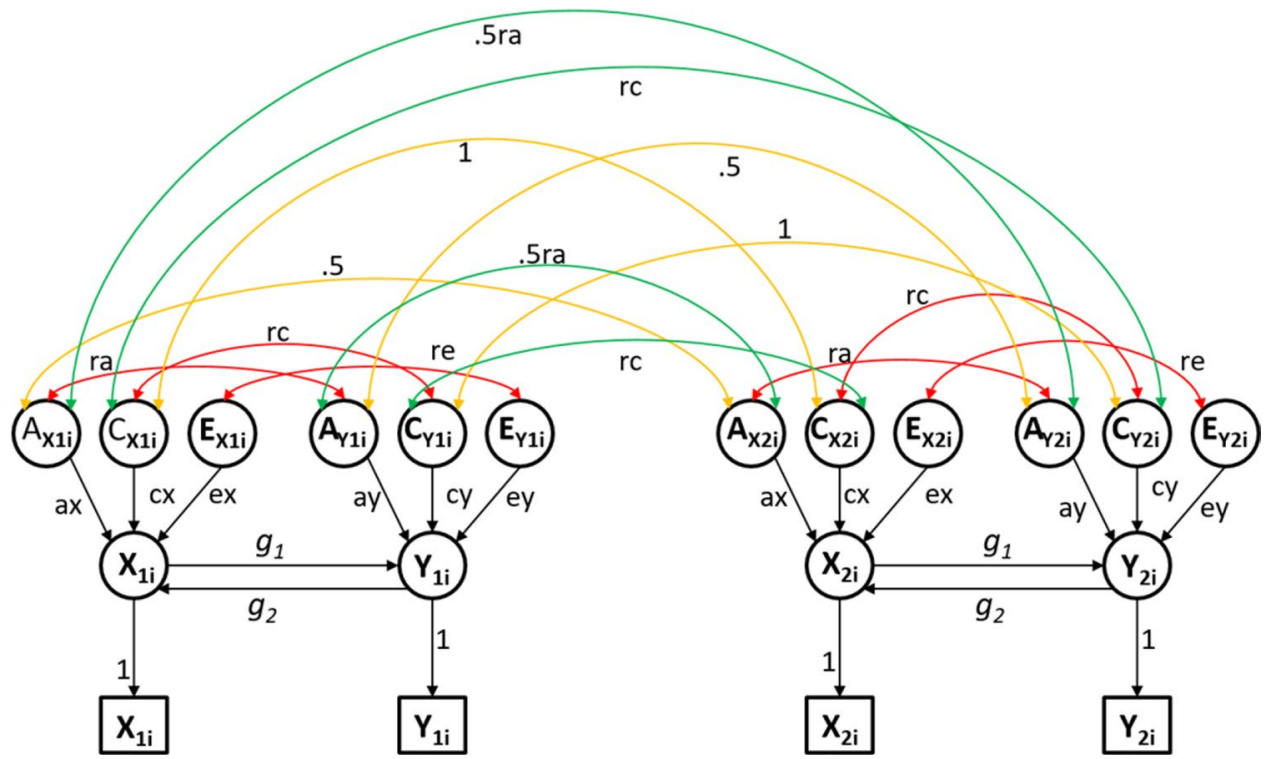

Fig. 1 Path diagram representing the Direction of Causation (DoC) twin model given two traits: variable $X$ and variable $Y$ measured in dizygotic (DZ) twins ( $\operatorname{twin}_{1}$ and $\operatorname{twin}_{2}$ ). Squares represent observed variables, while circles represent latent variables. $A, C$ and $E$ stand for additive genetic effects, shared and unique environmental effects, respectively. The double headed arrows represent within/between twins covariances of additive genetic effects (ra), shared environmental effects (rc) and unique environmental effects (re). The cross-twin

for pleiotropy, the approach facilitates the use of PGS as instruments; and (3) in specific circumstances, the approach confers substantial gains in power relative to the standard MR approaches.

\section{Methods}

Direction of Causation (DoC) twin model was advanced as an exploratory approach to establish direction of causation between two correlated traits (Heath et al. 1993; Gillespie et al. 2003; Verhulst et al. 2012; Verhulst and Estabrook 2012). In contrast, MR (Smith and Hemani 2014; Burgess and Thompson 2015) is used to test unidirectional causation (from designated causal exposure to outcome). ${ }^{1}$ Here we propose the MR-DoC twin model, developed by imposing restrictions on the DoC parameters to represent unidirectional causality hypotheses, and by extending the model to include measured genetic variants as instrumental variables.

\footnotetext{
${ }^{1}$ Bidirectional MR, as presented in the literature, involves two unidirectional sequential tests: Model 1 representing the hypothesis1 $\mathrm{X}$ causes $\mathrm{Y}$ (parameter $\mathrm{g}_{1}$ estimated), and Model 2 representing the hypothesis $2 \mathrm{Y}$ causes $\mathrm{X}$ (parameter $\mathrm{g}_{2}$ estimated, see Fig. 1 below). A true bidirectionality test involves estimating simultaneously the $\mathrm{g}_{1}$ and $\mathrm{g}_{2}$ parameters.
}

covariance between additive genetic effects is fixed to .5 (1) for DZ (MZ) twins. DZ (MZ) twins are expected to share on average 50\% $(100 \%)$ of the genetic effects underlying both traits, hence the crosstwin cross-trait covariance is fixed to .5(1) ra for DZ (MZ) twins. Single headed arrows represent causal effects. Note, the model as depicted, is not identified. Typically ra, rc, and re are assumed to be zero in the application of the DoC twin model

Integrating MR with DoC allows us to test certain MR assumptions.

\section{The direction of causation twin model}

The Direction of Causation (DoC) twin model (Heath et al. 1993; Gillespie et al. 2003; Verhulst et al. 2012; Verhulst and Estabrook 2012) uses cross-sectional data observed in monozygotic (MZ) and dizygotic (DZ) twins to test causal hypotheses regarding the association between two traits. In contrast to MR, DoC does not necessarily involve a prior hypothesis concerning the causal direction. The path diagram of such a model is shown in Fig. 1, given an exposure variable $\mathrm{X}$ and an outcome variable $\mathrm{Y}$, observed in $\mathrm{DZ}$ twins.

In Fig. $1 \mathrm{X}$ and $\mathrm{Y}$ are mutually causally related (parameters $g_{1}$ and $g_{2}$ ). The traits are subjected to the influence of latent additive genetic $\left(A_{X}\right.$ and $\left.A_{Y}\right)$, shared $\left(C_{X}\right.$ and $\left.C_{Y}\right)$ and unique $\left(E_{X}\right.$ and $\left.E_{Y}\right)$ environmental effects, influences which can be direct or indirect, i.e., via the causal paths $\left(\mathrm{g}_{1}\right.$ and $g_{2}$ ). As an instance of CTD, this model has the usual assumptions concerning random mating and the absence of genotype-environment interplay $(\mathrm{G} \times \mathrm{E}$ interaction, $\mathrm{G} \times \mathrm{E}$ covariance). The cross-twin correlation of the shared environmental variables is assumed to equal 1 within-trait, and $r c$ across traits, regardless of zygosity. By definition, the 
cross-twin correlation of unique environmental effects is fixed to zero both within and across traits.

The model as depicted in Fig. 1 is not identified; it requires additional restrictions to identify the parameters. By imposing restrictions on the parameters, one can model several alternative hypotheses concerning the observational association between $\mathrm{X}$ and $\mathrm{Y}$. The 'tertium quid' hypothesis, that a third variable causes both traits, can be tested by constraining the parameters $g_{1}$ and $g_{2}$ to equal zero (i.e., the saturated bivariate model). Uni- and bidirectional causal hypotheses can be tested by fixing to zero the within- and cross-twin cross-trait genetic and environmental correlations (ra, rc, re), and estimating the causal parameters $\mathrm{g}_{1}$ and/ or $g_{2}$ (i.e., the uni- and the bidirectional causality models). These competing and nested alternative hypotheses can be tested by likelihood ratio (Duffy and Martin 1994; Heath et al. 1993), provided: (1) the two traits differ in their sources of variance (Heath et al. 1993); and (2) there are at least three sources of variance influencing the traits (i.e., A, E, and either $\mathrm{C}$ or $\mathrm{D}$, where $\mathrm{D}$ denotes dominance) (Mather and Jinks 2012). Given the assumptions mentioned above, we have (Heath et al. 1993):

$X_{i j}=a_{X} A_{X i j}+c_{X} C_{X i j}+e_{X} E_{X i j}+g_{2} Y_{i j}$

$\mathrm{Y}_{\mathrm{ij}}=\mathrm{a}_{\mathrm{Y}} \mathrm{A}_{\mathrm{Yij}}+\mathrm{c}_{\mathrm{Y}} \mathrm{C}_{\mathrm{Yij}}+\mathrm{e}_{\mathrm{Y}} \mathrm{E}_{\mathrm{Yij}}+\mathrm{g}_{1} \mathrm{X}_{\mathrm{ij}}$

where subscript $i$ stands for twin pair, and $j$ for twin $(j=1$, 2 ); $\mathrm{X}$ is the exposure variable, and $\mathrm{Y}$ is the outcome variable; a, c and e represent the path coefficients relating the phenotypes ( $\mathrm{X}$ and $\mathrm{Y}$ ) to the $\mathrm{A}, \mathrm{C}$, and $\mathrm{E}$, respectively. The parameters $\mathrm{g}_{1}$ and $\mathrm{g}_{2}$ are the causal paths accommodating the direct causal effects of $\mathrm{X}$ on $\mathrm{Y}\left(\mathrm{g}_{2}\right)$ and $\mathrm{Y}$ on $\mathrm{X}\left(\mathrm{g}_{2}\right)$.

\section{Standard Mendelian randomization}

The MR model is an instrumental variable regression model, which employs genetic variants as instrumental variables to test causal hypotheses regarding the association between an exposure and an outcome (Smith and Hemani 2014; Burgess and Thompson 2015). Here we assume that the instrument is a polygenic score (PGS). Three assumptions must hold for a genetic variant to be a valid instrumental variable, as shown in Fig. 2. Assumption 1: The genetic instrument (PGS) is robustly associated with the exposure variable $X\left(b_{1} \neq 0\right.$ in Fig. 2); Assumption 2: PGS is independent of confounders $\mathrm{C}(\mathrm{m}=0$; PGS $\perp \mathrm{C})$; Assumption 3: PGS is independent of the outcome variable $\mathrm{Y}$ conditional on the exposure $\mathrm{X}$ and confounders $\mathrm{C}\left(\mathrm{b}_{2}=0\right.$; PGS $\left.\perp \mathrm{Y} \mid \mathrm{X}, \mathrm{C}\right)$.

In MR, the third assumption pertains to possible pleiotropic effects of the instrument (PGS), or to the likelihood of including variant(s) in linkage disequilibrium with variants affecting the outcome. In practice, the 'no pleiotropy' assumption may be violated, particularly when

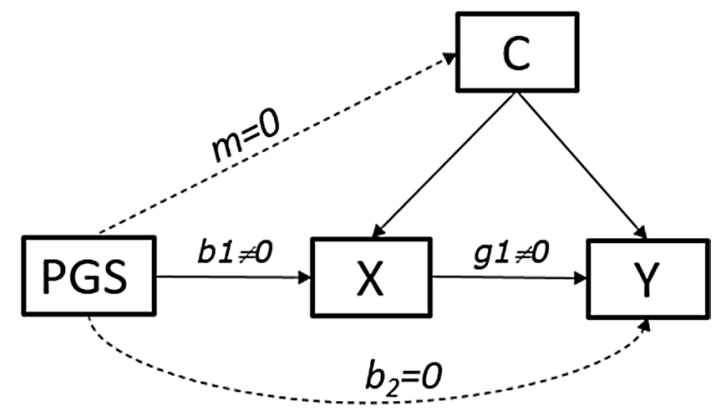

Fig. 2 Assumptions in Mendelian randomization. Hypothesis: $\mathrm{X}$ causes $\mathrm{Y}$. By assumption, the regression coefficients ( $\mathrm{m}$ and $\mathrm{b}_{2}$ ) associated with the dashed lines are zero. $P G S$-polygenic score, $X$-exposure variable, $Y$-outcome variable, $C$-confounders

the instrument is a PGS combining the effects of multiple genetic variants (note that a single variant with pleiotropic effects in principle renders the polygenic score invalid as an instrumental variable). This core MR assumption is illustrated in Fig. 3 where we consider several MR models with and without pleiotropic instruments, and pinpoint the definition of the no pleiotropy assumption.

Among the methods of causal effect estimation in the standard MR are the two-stage least squares (2SLS) and the ratio of coefficients methods. In 2SLS, first, the instrumental variable (e.g., the polygenic score) is used to predict the exposure $\mathrm{X}$, and second, the outcome is regressed on the predicted values of $X$. In the ratio of coefficients method, the causal effect is computed as a ratio of regression coefficients, with the numerator obtained in the regression of the outcome on the instrument, and the denominator obtained in the regression of the exposure on the instrument [see (Burgess and Thompson 2015)]. Both methods are based on least squares estimation, and are expected to yield equivalent results in MR studies involving a single instrumental variable (Burgess and Thompson 2015).

The standard MR model can also be fitted in a single step as a structural equation model as depicted in Fig. 3 (panel C), with maximum likelihood (ML) estimation [see (Kohler et al. 2011)]. The causal parameter $\widehat{g}_{1}$ in Fig. $3 \mathrm{c}$ can be tested by the means of a likelihood ratio or a Wald test.

\section{Integrating Mendelian Randomization with the Direction of Causation twin model (the MR-DoC model)}

In observational studies, the Mendelian Randomization and the Direction of Causation twin model offer some traction in testing a hypothesized direction of causality. As demonstrated below, the combined MR-DoC model has definite advantages over the individual approaches, in terms of power and assumptions. Figure 4 displays a path diagram 
A

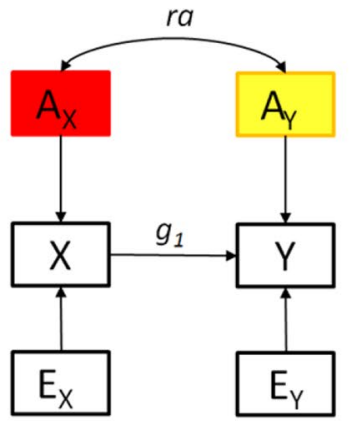

C

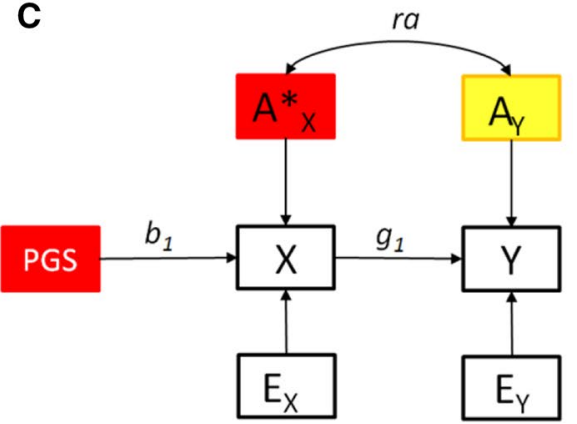

B
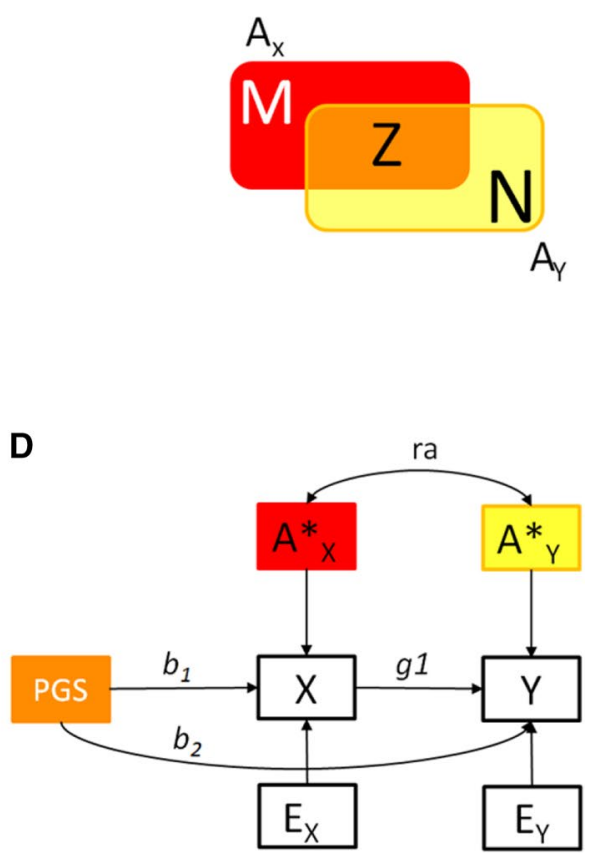

disequilibrium; C The MR model with a polygenic instrument (PGS) and 'no pleiotropy'. PGS is associated with X (parameter b1), but is assumed to have no direct influence on the outcome Y. This model holds only if the instrument PGS is constructed on the basis of a subset of variants from set $\mathrm{M}$. In the presence of PGS, $\mathrm{A}^{*}{ }_{\mathrm{X}}$ is a residual (in the regression of $X$ on PGS). D MR with pleiotropic genetic instrument. In this model, the PGS is constructed on the basis of a sample of genetic variants taken from set $Z$. The parameter $b_{2}$ accommodates the fact that the set of variants used to construct PGS underlies the variance of both $A_{X}$ and $A_{Y}$. The no pleiotropy assumption implies $b_{2}=0$
Fig. 4 Path diagrammatic representation of the MR-DoC model in $\mathrm{DZ}$ twins. The parameter $\mathrm{x}$ equals the standard deviation of the observed instrument, i.e., PGS in the circle is standardized. The model as depicted is not identified (see Table 1)

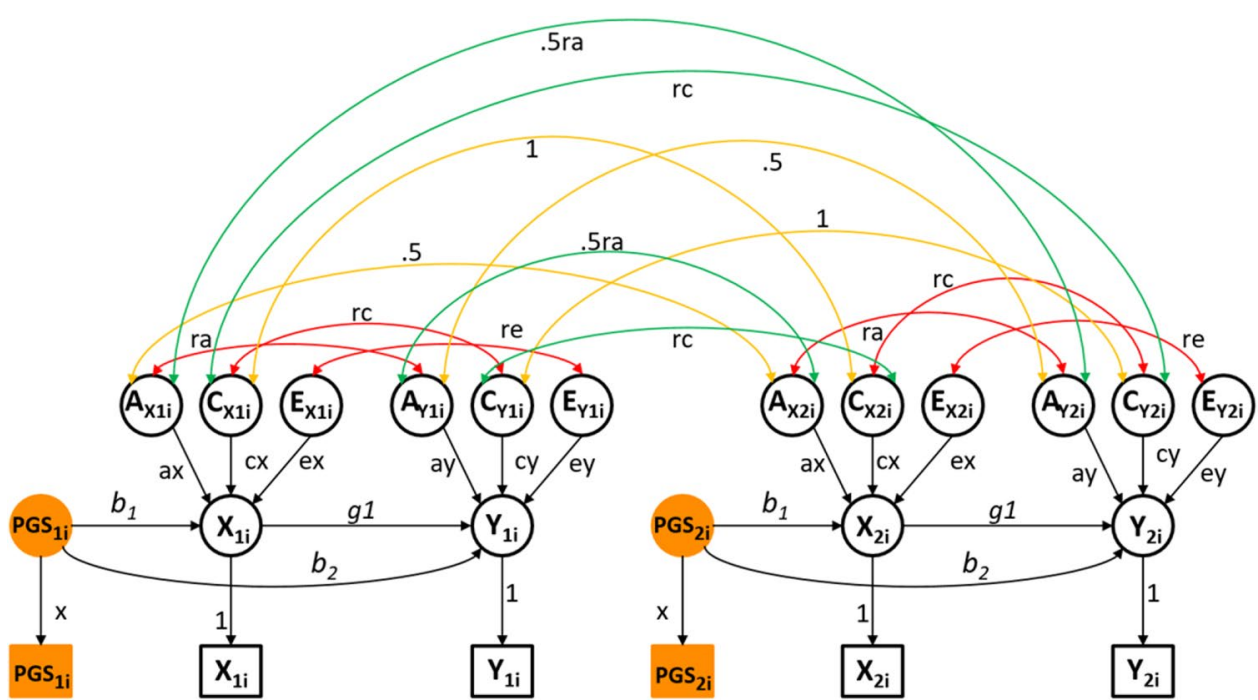


Table 1 Parameter constraints that render identified the model in Fig. 4

\begin{tabular}{|c|c|c|c|c|c|c|c|c|c|c|c|c|c|c|}
\hline Model & $\mathrm{x}$ & $a_{X}$ & $c_{X}$ & $e_{X}$ & $a_{Y}$ & $c_{Y}$ & $\mathrm{e}_{\mathrm{Y}}$ & ra & $\mathrm{rc}$ & re & $b_{1}$ & $b_{2}$ & $\mathrm{~g}_{1}$ & Identified? \\
\hline 1 & fr & fr & fr & fr & $\mathrm{fr}$ & fr & $\mathrm{fr}$ & fr & fr & fr & $\mathrm{fr}$ & fr & fr & No \\
\hline 2 & fr & $\mathrm{fr}$ & $\mathrm{fr}$ & fr & $\mathrm{fr}$ & fr & fr & fr & fr & 0 & fr & fr & $\mathrm{fr}$ & Yes \\
\hline 3 & $\mathrm{fr}$ & $\mathrm{fr}$ & fr & fr & $\mathrm{fr}$ & fr & fr & fr & fr & fr & $\mathrm{fr}$ & 0 & $\mathrm{fr}$ & Yes \\
\hline 4 & $\mathrm{fr}$ & $\mathrm{fr}$ & $\mathrm{fr}$ & $\mathrm{fr}$ & $\mathrm{fr}$ & $\mathrm{fr}$ & fr & fr & 0 & fr & $\mathrm{fr}$ & fr & $\mathrm{fr}$ & Yes \\
\hline 5 & $\mathrm{fr}$ & $\mathrm{fr}$ & 0 & fr & $\mathrm{fr}$ & 0 & fr & fr & 0 & $\mathrm{fr}$ & $\mathrm{fr}$ & $\mathrm{fr}$ & $\mathrm{fr}$ & No \\
\hline 6 & $\mathrm{fr}$ & $\mathrm{fr}$ & $\mathrm{fr}$ & fr & $\mathrm{fr}$ & 0 & $\mathrm{fr}$ & fr & 0 & $\mathrm{fr}$ & $\mathrm{fr}$ & $\mathrm{fr}$ & fr & Yes \\
\hline 7 & fr & fr & 0 & fr & fr & fr & fr & fr & 0 & fr & fr & fr & fr & No \\
\hline
\end{tabular}

Note 'fr' indicates that the parameter is estimated, ' 0 '一-that the parameter is constrained to equal 0 of the MR-DoC model. Note that, the model as depicted is not identified. We consider the issue of identification below.

The MR-DoC model is based on the following regression model:

$X_{i j}=a_{X} A_{X i j}+c_{X} C_{X i j}+e_{X} E_{X i j}+b_{1} P S_{i j}$

$Y_{i j}=a_{Y} A_{Y i j}+c_{Y} C_{Y i j}+e_{Y} E_{Y i j}+g_{1} X_{i j}+b_{2} P S_{i j}$

where $i$ stands for twin pairs, and $j$ for twin $(j=1,2)$. The vector of parameters is $\boldsymbol{\theta}=\left[\mathrm{ra}, \mathrm{rc}, \mathrm{re}, \mathrm{a}_{\mathrm{x}}, \mathrm{c}_{\mathrm{x}}, \mathrm{e}_{\mathrm{x}}, \mathrm{a}_{\mathrm{y}}, \mathrm{c}_{\mathrm{y}}, \mathrm{e}_{\mathrm{y}}, \mathrm{g}_{1}\right.$, $\left.\mathrm{b}_{1}, \mathrm{~b}_{2}, \mathrm{x}\right]$, where $\mathrm{x}$ is the standard deviation of the PGS. Here, and in Fig. 3, the parameter of interest is $g_{1}$, as it concerns the causal effect of exposure $\mathrm{X}$ on outcome Y. Note that the MR-DoC model includes the parameter $b_{2}$, representing the pleiotropic effects of PGS on Y (which are usually assumed to be absent, i.e., the central assumption of standard MR of 'no horizontal pleiotropy'). That is, the parameter $b_{2}$ represents the direct effect of the PGS on the outcome, and accounts for pleiotropy. The estimate of the causal effect $\mathrm{g}_{1}$ is unbiased provided the parameter $\mathrm{b}_{2}$ is included in the model. Where we refer to a pleiotropic instrument below, we mean that the parameter $b_{2}$ is not zero (parameter $b_{2} \neq 0$ ). Using ML estimation, we can test hypotheses concerning the parameters by means of a likelihood ratio or Wald test.

\section{Model identification}

Model identification concerns the question whether the observed data provide sufficient information to yield unique estimates of the unknown parameters collected in the vector $\boldsymbol{\theta}$ (Bollen and Bauldry 2010). In the present case, the observed information is summarized in the MZ and DZ $(6 \times 6)$ covariance matrices. We assume that the phenotypic means are equal in the $\mathrm{MZ}$ and $\mathrm{DZ}$ and are equal in the twin 1 and twin 2 members (this is obviously testable). As the parameterization of the means has no bearing on the identification of the covariance structure model, we do not consider them in addressing identification.

Local identification is evaluated at a given set of parameters, say $\boldsymbol{\theta}_{\mathrm{a}}$ and implies there are no points in the vicinity of point $\boldsymbol{\theta}_{\mathrm{a}}$ in the parameter space leading to the same expected covariance matrices $\Sigma_{\mathrm{MZ}}\left(\boldsymbol{\theta}_{\mathrm{a}}\right)$ and $\Sigma_{\mathrm{DZ}}\left(\boldsymbol{\theta}_{\mathrm{a}}\right)$ (Bollen and Bauldry 2010; Bekker et al. 2014). We evaluated local identification using symbolic algebra in Maple (Morgan et al. 2005). Derks et al. (Derks et al. 2006) previously used this method in the context of twin modeling. Using Maple, we checked whether the rank of the Jacobian matrix is full column rank. The Jacobian matrix contains the first order derivatives of the elements in the matrices $\Sigma_{\mathrm{MZ}}\left(\boldsymbol{\theta}_{\mathrm{a}}\right)$ and $\Sigma_{\mathrm{DZ}}\left(\boldsymbol{\theta}_{\mathrm{a}}\right)$ with respect to the free parameters. If the Jacobian is not full column rank, we require additional parameter constraints (on the elements in the parameter vector $\boldsymbol{\theta}_{\mathbf{a}}$ ). Having established local identification in this manner, we proceeded to address the question of resolution by considering the statistical power to estimate the parameters of interest.

We considered identification in seven models given in Table 1. Model 1, in which all parameters are estimated freely, is not identified. However, constraining to zero any of the parameters re (Model 2), $b_{2}$ (Model 3) or rc (Model 4) renders the model identified. All parameters are identified if the two traits differ with respect to their ACE model. This is the case when the exposure is an ACE trait and the outcome (conditional on the exposure) is an AE trait (implying the parameters $\mathrm{c}_{\mathrm{y}}$ and $\mathrm{rc}$ equal zero, Model 6). Note that in this case $\mathrm{Y}$ (unconditional) is characterized by shared environmental effects on $\mathrm{Y}$ (transmitted from $\mathrm{C}_{\mathrm{X}}$, through the $\mathrm{g}_{1}$ path) (De Moor et al. 2008). That is, part of variation in $Y$ is attributable to shared environmental factors that contribute to $\mathrm{X}$. These shared environmental effects specific to the exposure identify the parameter $\mathrm{g}_{1}$ as they can be related with Y only via the causal path. Furthermore, we found that MR-DoC is not identified when the traits' variances are limited to two sources (e.g., $\mathrm{X}$ and $\mathrm{Y}$ are both $\mathrm{AE}$ traits, Model 5), or when the exposure is an $\mathrm{AE}$ trait and the outcome is an ACE trait (Model 7). Fixing the parameter re to 0 is a constraint commonly employed in the MZ twin intra-pair differences model (Kohler et al. 2011) and in the discordant twin design. In other words, it is assumed that there is no latent confounding from unique environmental sources. That is, the unique environmental component influencing the exposure, influences the outcome only via its effect on the exposure, 

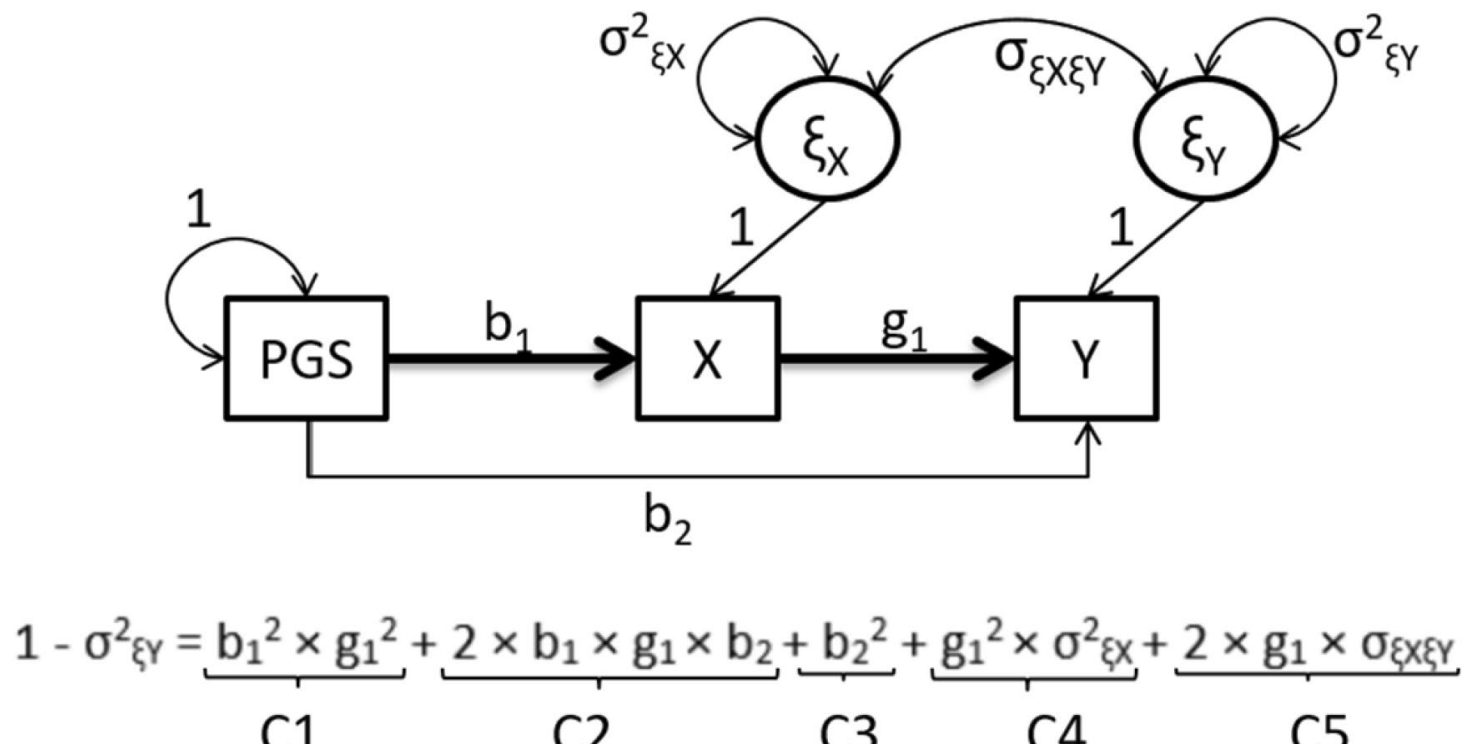

Fig. 5 Effect size calculation for the power analyses. $P G S$-polygenic score, $X$-exposure variable, $Y$-outcome variable, $\xi_{X^{-}}$residual $\mathrm{X}, \sigma_{\xi X^{-}}^{2}$ variance in the exposure, not explained by the instrumental variable PGS, $\xi_{Y}$-residual $\mathrm{Y}, \sigma^{2} \xi_{Y}$-variance in $\mathrm{Y}$ not explained by the MR model, $\sigma_{\xi X \xi Y}$ the covariance between $\xi_{\mathrm{X}}$ and $\xi_{\mathrm{Y}}, b_{1}$-regression coefficient in the MR regression of the exposure $\mathrm{X}$ on the instrument, $b_{2}$-regression coefficient in the MR regression of the outcome on the

but not directly. The following results are based on the model with this identifying constraint in place, i.e., re $=0$.

\section{Power calculations and the type I error rates}

We used exact data simulation to create data that fit a given identified model exactly [i.e., the observed covariance matrices equalled the expected covariance matrices exactly; see (Van Der Sluis et al. 2008)]. That is, we simulated normal data on the exposure, outcome and polygenic scores in 2000 MZ and DZ twin pairs, given the scenarios described in the Supplementary Tables S1 and S6. The polygenic score was generated as a continuous, normal variable. We considered PGS with effect sizes of 5 and $10 \%$ explained variance of the exposure. We fitted the true model, thus retrieving the parameter values exactly as specified, and then dropped the parameters of interest to assess the power in the standard way using the non-central $\chi^{2}$ distribution, with noncentrality parameter (NCP) $\lambda$. We adopted an alpha of .05 throughout. Data were simulated in R using the MASS library function mvrnorm() with the empirical = TRUE option (R-Core-Team 2015). The MR-DoC model was fitted to the population covariance matrices in OpenMx (Neale et al. 2016). We used the R-package AER (Kleiber and Zeileis 2008) to conduct 2SLS estimation in the standard MR using the sample of twin 1 data, effectively a sample of unrelated individuals. We used the mRnd power calculator to calculate the power
PGS, $g_{l}$-the causal effect of X on Y. Note that all parameters are estimated simultaneously in this MR model, hence e.g., the $b_{2}$ parameter estimated in the MR regression will not equal the regression coefficient obtained in the OLS regression of Y on PGS (the latter regression coefficient captures both direct and indirect effect of the PGS on Y)

of the 2SLS procedure (Brion et al. 2013). We chose effect sizes by considering the decomposition of the variance of the outcome Y, as illustrated in Fig. 5. That is, given that the outcome $\mathrm{Y}$ is standardized $\left(\sigma_{Y}^{2}=1\right)$, we considered the components making up the explained variance, i.e., $1-\sigma^{2} \xi_{\mathrm{Y}}$, as a function of the regression parameters $b_{1}, b_{2}, g_{1}$, the variances of the residuals $\xi_{X}$ and $\xi_{Y}$ (parameters $\sigma_{\xi X}^{2}$ and $\sigma_{\xi \mathrm{Y}}^{2}$ ), and the covariance between the residuals $\xi_{\mathrm{X}}$ and $\xi_{\mathrm{Y}}$ (parameter $\sigma_{\xi \mathrm{X} \xi \mathrm{Y}}$ ).

We varied (a) the strength of the instrumental variable, defined as the percentage of variance explained in the exposure $X$ by instrumental PGS; (b) the variance of $\xi_{X}\left(\right.$ residual X), i.e., parameter $\sigma_{\xi X}^{2}$, representing the percentage of variance in the exposure, not explained by the instrumental variable; (c) the variance in $\xi_{\mathrm{Y}}$ (residual Y), i.e., parameter $\sigma^{2} \xi_{Y}$, representing the percent of variance in Y not explained by the MR model; and (d) the covariance between $\xi_{\mathrm{X}}$ and $\xi_{\mathrm{Y}}$ (parameter $\sigma_{\xi \mathrm{X} \xi \mathrm{Y}}$ ). Using the path tracing rules, we can distinguish five components of variance (C1 to C5, Fig. 5) that involve the parameters of interest $g_{1}$ (the causal effect) and $b_{2}$ (representing the direct-pleiotropic-effect of the instrument on the outcome). In all scenarios, the total explained variance of the outcome $\mathrm{Y}$ equalled $10 \%$. The parameter values used in simulations are included in the Supplementary Tables S1 and S6. To provide an indication on the potential gains in power conferred by our approach relative to a standard MR analysis 
Table 2 Results of the comparison of the standard MR in analysis of unrelated individuals and MR-DoC twin model

\begin{tabular}{|c|c|c|c|}
\hline Scenario & $\begin{array}{l}\text { Parameter } \\
\text { values (simu- } \\
\text { lated) }\end{array}$ & $\begin{array}{l}\text { TSLS/Two-sample MR/ } \\
\text { MR as SEM (estimated) }\end{array}$ & MR-DoC (estimated) \\
\hline $\begin{array}{l}\text { 1. Non-pleiotropic instrument and non-zero causal relationship between expo- } \\
\text { sure and outcome }\end{array}$ & $\begin{array}{l}\mathrm{b}_{1}=0.3162 \\
\mathrm{~b}_{2}=0 \\
\mathrm{~g}_{1}=0.1838\end{array}$ & $\begin{array}{l}\mathrm{b}_{1}=0.3162 \\
\mathrm{~g}_{1}=0.1838\end{array}$ & $\begin{array}{l}\mathrm{b}_{1}=0.3162 \\
\mathrm{~b}_{2}=0 \\
\mathrm{~g}_{1}=0.1838\end{array}$ \\
\hline $\begin{array}{l}\text { 2. Pleiotropic instrument and non-zero causal relationship between exposure } \\
\text { and outcome }\end{array}$ & $\begin{array}{l}\mathrm{b}_{1}=0.3162 \\
\mathrm{~b}_{2}=0.1599 \\
\mathrm{~g}_{1}=0.1265\end{array}$ & $\begin{array}{l}\mathrm{b}_{1}=0.3162 \\
g_{1}=0.6324\end{array}$ & $\begin{array}{l}\mathrm{b}_{1}=0.3162 \\
\mathrm{~b}_{2}=0.1599 \\
\mathrm{~g}_{1}=0.1265\end{array}$ \\
\hline $\begin{array}{l}\text { 3. Pleiotropic instrument and no causal relationship between exposure and } \\
\text { outcome }\end{array}$ & $\begin{array}{l}\mathrm{b}_{1}=0.3162 \\
\mathrm{~b}_{2}=0.3162 \\
\mathrm{~g}_{1}=0\end{array}$ & $\begin{array}{l}\mathrm{b}_{1}=0.3162 \\
g_{1}=1.00\end{array}$ & $\begin{array}{l}\mathrm{b}_{1}=0.3162 \\
\mathrm{~b}_{2}=0.3162 \\
\mathrm{~g}_{1}=-4.86 \mathrm{e}-08\end{array}$ \\
\hline
\end{tabular}

Additive genetic (A), shared environmental (C) and unique environmental (E) effects contributed to the variance of both the exposure $\left(\mathrm{a}^{2}{ }_{\mathrm{X}}=0.5\right.$, $\left.\mathrm{c}^{2}{ }_{\mathrm{X}}=0.2, \mathrm{e}_{\mathrm{X}}^{2}=0.3\right)$ and the outcome $\left(\mathrm{a}^{2}{ }_{\mathrm{X}}=0.2, \mathrm{c}^{2}{ }_{\mathrm{X}}=0.1, \mathrm{e}_{\mathrm{X}}^{2}=0.7\right)$ variable, and genetic and shared environmental factors contributed to the correlations between the traits $\left(\mathrm{r}_{\xi \mathrm{X} \xi \mathrm{Y}}=0.2\right)$. Incorrectly estimated parameter values are in italics

of data obtained in unrelated individuals, we report the number of unrelated persons required to attain equivalent power as MR-DoC based on 2000 twin pairs.

We used Monte Carlo Simulations to evaluate type I error rate across all scenarios considered above. We simulated 10,000 samples under the null model of no causal effect of the exposure on the outcome variable (parameter $\mathrm{g}_{1}$ equalled 0). Each sample consisted of 2000 twin pairs. The type I error was calculated as the percentage of datasets in which the null hypothesis was incorrectly rejected given two significance thresholds, 0.05 and 0.01 .

\section{Results}

\section{Parameter recovery in standard MR and MR-DoC with valid or invalid (i.e., pleiotropic) instrumental variables (given re $=0$ )}

Table 2 displays results obtained using non-pleiotropic (i.e., Fig. $3, b_{2}=0$ ), or pleiotropic (i.e., $b_{2} \neq 0$, see Fig. 4 ) instrumental variables in the causal effect estimation. Given $b_{2}=0$, results indicate that all estimation methods recover the true parameter value (scenario $S 1$, Table 2). As is to be expected, $b_{2} \neq 0$ leads to biased estimates of the causal effect when employing standard MR methods (e.g., 2SLS or ratio of coefficients). MR-DoC recovers correctly the true parameter values (scenario S2, Table 2). Finally, we checked parameter recovery when the instrument has pleiotropic effects, but there is no causal effect of the exposure $\mathrm{X}$ on outcome $\mathrm{Y}$ (i.e., $\mathrm{b}_{2} \neq 0$ and $\mathrm{g}_{1}=0$; scenario $\mathrm{S} 3$, Table 2). Results showed that standard MR detects a causal effect (when in truth there is none), while MR-DoC does not. We remind the reader that we have set re $=0$ to obtain these results.

\section{Power and type I error rates}

Using Monte Carlo simulations we established that the type I error rate is correct (see Supplementary Tables S2, S4 and S7). Figure 6 (and Supplementary Tables S3, S5 and S8) displays the results pertaining to the power to detect the causal effect in standard MR and in MR-DoC.

With a valid instrumental variable (no pleiotropy) and all parameters freely estimated (including the parameter re, Table S3), the main factors that influence MR-DoC's power are: (a) instrument's strength; (b) the genetic covariance structure of $\mathrm{X}$ (exposure) and $\mathrm{Y}$ (outcome); and (c) the magnitude of the residual $\mathrm{X}-\mathrm{Y}$ correlation. As expected, increasing instrument's strength increases power. For instance, with an ACE trait as the exposure variable $\left(\mathrm{a}^{2}{ }_{\mathrm{X}}=.5, \mathrm{c}^{2}{ }_{\mathrm{X}}=.2\right.$, $\mathrm{e}^{2}{ }_{X}=.3$ ), an outcome variable having roughly the same mode of inheritance $\left(\mathrm{a}^{2}{ }_{\mathrm{Y}}=.5, \mathrm{c}^{2}{ }_{\mathrm{Y}}=.2, \mathrm{e}_{\mathrm{Y}}^{2}=.3\right)$, and a residual correlation of $\mathrm{r}_{\xi \mathrm{X} \xi \mathrm{Y}}=.2$, the power of the MR-DoC equals .627 and .905 when the instrument explains 5 and $10 \%$ of the variance in the exposure, respectively. However, contrary to the standard DoC literature, having traits with similar genetic covariance structure has no bearing on MRDoC's power to detect the causal effect. This is the case for instance, if both the outcome and exposure are ACE traits. Power is the highest when the outcome variable has a lower heritability than the exposure variable. For example, power increases from .622 in Scenario S1G (with a 50\% heritable outcome and a $20 \%$ heritable exposure) to .658 in Scenario S1I (with a 20\% heritable outcome variable and a 50\% heritable exposure; Table S3). Finally, increasing $\mathrm{X}-\mathrm{Y}$ residual correlation reduces MR-DoC's power. For instance, with an outcome and an exposure having roughly the same mode of inheritance, and an instrument explaining 5\% of the variance in the exposure, MR-DoC's power drops from .627 (Scenario S1H, Table S3) to .312 (Scenario S1B) when the residual correlation increases from $r_{\xi \mathrm{X} \xi \mathrm{Y}}=0.2$ to $r_{\xi \mathrm{X} \xi \mathrm{Y}}=0.4$. 

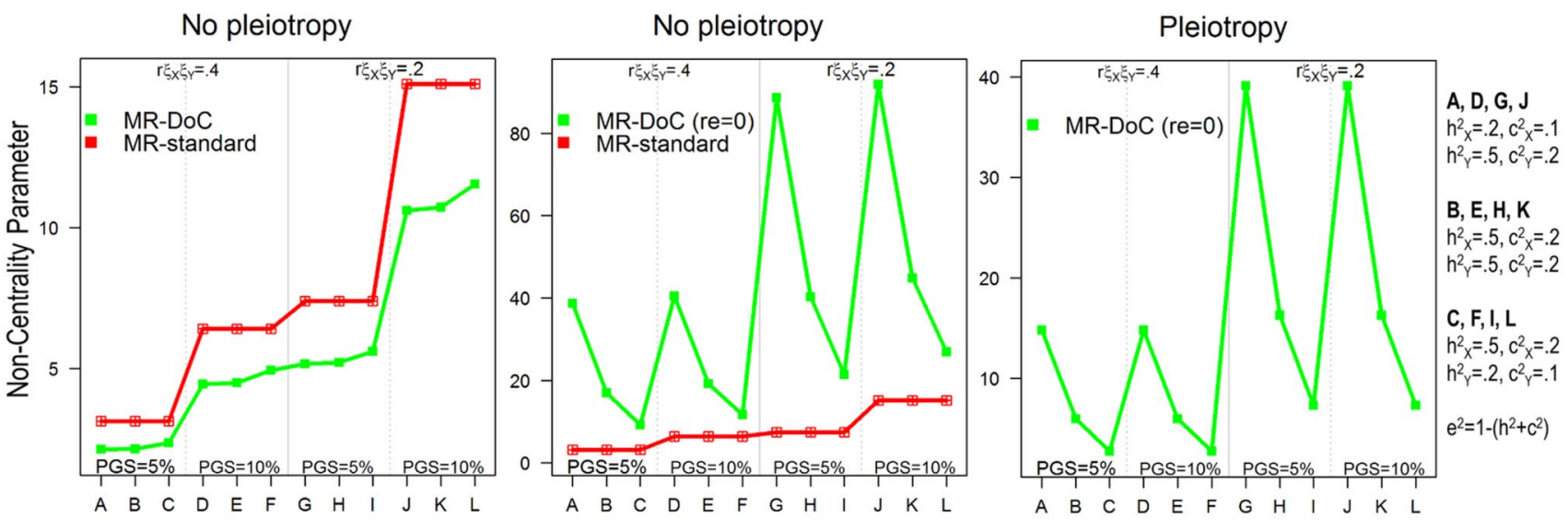

Fig. 6 Results given a non-pleiotropic instrument (parameter $b_{2}=0$; left and middle panels), and given a pleiotropic instrument (parameter $b_{2} \neq 0$; right panel). Fitting the model with the parameter $g_{1}$ freely estimated, and with the parameter $\mathrm{g}_{1}$ constrained to equal zero provided the Non-Centrality Parameter (NCP). The standard MR is

Given equal N, standard MR (4000 unrelateds) has larger power than MR-DoC (4000 twins) only when $b_{2}=0$ and re $\neq 0$ (Fig. 6, left). Yet, assuming data collected in twins are readily available, greater statistical power is available in the MR-DoC model (than from reducing the twin pairs to singletons and resorting to standard MR, Table S5).

We also calculated the required $\mathrm{N}$ of unrelated individuals to achieve the same power as 2000 twin pairs (Table 3). We found the yield of MR-DoC substantial if the parameter re was fixed to zero (as simulated, Fig. 6, middle). For example, with a sample of 2000 twin pairs, MR-DoC yields a NCP $\lambda$ of 38.68 given an instrument explaining $10 \%$ of the variance in the exposure, a residual correlation of $\mathrm{r}_{\xi X \xi Y}=0.2$, an exposure variable with low heritability $\left(\mathrm{a}^{2}{ }_{\mathrm{X}}=0.2, \mathrm{c}^{2}{ }_{\mathrm{X}}=0.1\right.$, $\left.\mathrm{e}^{2}{ }_{\mathrm{X}}=0.7\right)$, and a moderately heritable outcome $\left(\mathrm{a}^{2}{ }_{\mathrm{Y}}=0.5\right.$, $\left.\mathrm{c}^{2}{ }_{\mathrm{Y}}=0.2, \mathrm{e}_{\mathrm{Y}}^{2}=0.3\right)$. Standard MR needs about 56737 unrelated individuals to achieve equivalent power (Table 3).

Given $b_{2} \neq 0$ and re $=0$, MR-DoC's power increases with: (a) decreasing X-Y residual correlation, and (b) decreasing residual variance of the exposure (Fig. 6, right; see for details, Tables S2 and S5). Regarding the former, power is always larger when the association between the exposure and the outcome is largely causal in nature (i.e., when the residual correlation drops from $r_{\xi X \xi Y}=0.4$ to $r_{\xi X \xi Y}=0.2$ ). Regarding the latter, power is always greater in scenarios where the exposure has low heritability. For instance, the NCP $\lambda$ increases from 7.29 to 39.1 when the heritability of the exposure decreases from $50 \%$ (S2I) to $20 \%$ (S2J, Table S8). The same pattern of results was observed when $\mathrm{b}_{2}=0$ and re $=0$ (see Fig. 6, middle, and Table S5). Lowering the parameter $\mathrm{a}_{\mathrm{x}}$ (reducing the background additive genetic variance of the exposure) will reduce the residual variance in the regression of the exposure on the outcome based on two-stage least squares in a sample of 4000 unrelateds. The MR-DoC twin model used maximum likelihood and a sample of 2000 twin pairs. $r_{\xi X \xi Y}$ residual exposure-outcome correlation, $P G S$ polygenic score

and this increases the instrument strength. This will render the role of $g_{1}$ (the causal effect) in connecting $\mathrm{X}$ and $\mathrm{Y}$ relatively greater. This in turn increases the power to detect $g_{1}$. We note that the same applies when lowering the parameter $\mathrm{c}_{\mathrm{x}}$. Note that the instrument's strength has no longer a bearing on power when $b 2 \neq 0$, i.e., when it affects the outcome both directly (parameter $\mathrm{b}_{2}$ ) and indirectly, via the exposure (parameter $b_{1}$ ). For instance, 2000 twin pairs yield an NCP $\lambda$ of 5.98 given two traits having identical variance components $\left(\mathrm{a}^{2}{ }_{\mathrm{X}}=\mathrm{a}^{2}{ }_{\mathrm{Y}}=0.5 ; \mathrm{c}^{2}{ }_{\mathrm{X}}=\mathrm{c}^{2}{ }_{\mathrm{Y}}=0.2 ; \mathrm{e}^{2}{ }_{\mathrm{X}}=\mathrm{e}^{2}{ }_{\mathrm{Y}}=0.3\right)$, and a large residual correlation $\left(\mathrm{r}_{\xi \mathrm{X} \xi \mathrm{Y}}=0.4\right)$, regardless of

Table 3 The number of unrelated individuals $(\mathrm{N})$ required by MRstandard to achieve the same NCP as MR-DoC in 2000 twin pairs

\begin{tabular}{ll}
\hline Scenario (S1) & $\begin{array}{l}\text { N required by MR standard to achieve } \\
\text { the same NCP as MR-DoC with re=0 }\end{array}$ \\
\hline A & 56,737 \\
B & 24,945 \\
C & 13,472 \\
D & 28,515 \\
E & 13,480 \\
F & 8206 \\
G & 53,774 \\
H & 24,440 \\
I & 13,013 \\
J & 13,558 \\
K & 13,239 \\
L & 7954
\end{tabular}

The number of unrelateds was estimated based on the NCP obtained by fitting the standard MR as a structural equation model, with estimation of the causal effect based on maximum likelihood (similar to MR-DoC) 
whether the instrument explains 5 or $10 \%$ of the variance in the exposure (Scenarios S2B and S2E, Table S8). As mentioned above, contrary to the standard DoC literature [see e.g., (Heath et al. 1993)], MR-DoC does not require the exposure and the outcome variable to have radically different covariance structures to ensure sufficient power to test unidirectional causal hypotheses.

\section{Discussion}

Our aim was to integrate MR and the classical twin model to render testable the MR's strong assumption that the instrumental variable has no direct effect on the outcome, conditional on the exposure and confounders. We showed that, with standard MR methods, violations of this assumption readily lead to biased causal effect estimates, or may even yield spurious false positives. MR-DoC correctly detects causal effects and provides accurate parameter estimates even if the instrument is pleiotropic. With traits that have the same covariance structure (e.g., when both the outcome and exposure are ACE traits), the weaker assumption (used also in the MZ discordant twin design) that there is no latent confounding from unique environmental sources of variation may be needed (see Table 1). However, the effect of re $=0$ can be studied by fixing re to various values with the objective of gauging the sensitivity of the results to the value of re.

Note that the assumption re $=0$ is not required with traits that have different covariance structures (i.e., with an ACE trait as the exposure and an $\mathrm{AE}$ trait as an outcome one may estimate re, $b_{2}$ as well as $g_{1}$, see Fig. 4). We did not consider extensively this scenario because although formally identified, this model has poor resolution (but see Supplementary Table S9 and Figure S1 for results of a small power study given parameter $r_{c}=0$ ). That is, employing the model would require pooling the genotype data from multiple twin registries in a mega-analysis to ensure adequate power.

Aside from providing the means to relax the 'no pleiotropy' assumption, the MR-DoC twin model confers several other advantages in understanding causal relationships between exposures and outcomes. First, MR-DoC provides full statistical description of the observed exposure-outcome relationship, allowing one to disentangle the causal effect of the exposure on the outcome, from potential pleiotropic effects of the instrumental variable, as well as from the contribution of other genetic and environmental factors to the variances and the covariance of the two traits. Second, the twin data provide sufficient information to estimate the direct path from the instrument to the outcome (i.e., the parameter $b_{2}$ in Fig. 4). Importantly, this path captures not only pleiotropic effects, but also possible effects of other variants affecting the outcome which are in linkage disequilibrium with the instrument. Third, our approach opens up the possibility of using strong genetic instrumental variables in the form of polygenic scores. This is generally desirable in the standard MR design, as the strength of the valid instrumental variable has a bearing on the precision of the causal estimate [i.e., with weak instruments the estimate tends to approach the observed OLS exposure-outcome association (Bound et al. 1995)], and on the distribution of the causal effect estimate [the weaker the instrument, the more skewed the distribution; see Fig. 7.1 in (Burgess and Thompson 2015)]. As a consequence, significance tests and the construction of the confidence intervals, which rely on asymptotic normality, are no longer accurate. Consequently, tests may suffer inflated type I error rates (Burgess et al. 2015). In addition, strong instruments are desirable from the perspective of power, as our results showed [consistent with the literature, see, e.g., (Burgess et al. 2015)].

Interestingly, the strength of the instrument (i.e., defined in terms of percentage of explained variance in the exposure), has no bearing on the power when the instrument has pleiotropic effects, given that these effects are correctly modeled (in MR-DoC). The reason for this is that the exposure no longer features as a full mediator variable in the presence of a direct path between the instrument and the outcome. Correspondingly, the misfit due to dropping the causal parameter from the analysis is attenuated by the presence of the parameter $b_{2}$ in the model. That is, fixing the parameter $\mathrm{g}_{1}$ to zero will largely bias the parameter $b_{2}$, but will not affect the between twin covariance matrix (as is the case in the standard MR design where the sole path from the instrument to the outcome is via the exposure). Stated otherwise, the bias in $b_{2}$ will be greatest, leaving $\mathrm{ra}(\mathrm{Ax}, \mathrm{Ay})$ and $\mathrm{rc}$ $(\mathrm{Cx}, \mathrm{Cy})$ relatively unaffected, regardless of how strong the instrument is. In this circumstance, the power to detect the causal effect will primarily depend on the magnitude of the residual correlation between the two traits and, to a lesser extent, on their modes of inheritance.

An important caveat to consider when employing the MR-DoC model concerns the issue of measurement error. As extensively discussed in the DoC literature (Heath et al. 1993; Kohler et al. 2011) the estimates of the model parameters including the estimate of the parameter of interest $\mathrm{g}_{1}$ will be biased when there is measurement error in the exposure $\mathrm{X}$. This problem can be circumvented by employing multiple indicators of the exposure [i.e., see for details (Heath et al. 1993; Kohler et al. 2011)], or by including prior information on the reliability of the exposure.

MR-DoC is tailored for the readily available datasets collected worldwide on more than 1.5 million twins at the Twin and Family Registries [see (Hur and Craig 2013) for details on these rich resources of genotypic and phenotypic data]. We showed that twin data correctly detect causation and estimate effect sizes even in the presence of pleiotropy. Although 
primarily developed to address the 'no pleiotropy' assumption, our results demonstrate that MR-DoC has greater statistical power than standard MR analysis in unrelated individuals if the parameter re is zero (as fixed to zero in the model). With re fixed to zero, dropping the causal parameter $\mathrm{g}_{1}$ from the model greatly impacts all paths connecting the exposure and the outcome, both within and across twins (parameters ra and rc), creating a large discrepancy between the observed and the expected covariance matrix. This misfit is evident throughout the covariance matrix. Consequently, in some specific circumstances, testing causal hypotheses requires tens of thousands of unrelated individuals for one to achieve the same power as that conferred by several hundreds of twin pairs. It should be noted that unlike MR methods (Bowden et al. 2015, 2016) or asymmetry tests (Pickrell et al. 2016) based on summary statistics (methods which require GWAS results for both the exposure and the outcome variables), the approach proposed here only requires GWAS results for the exposure variable (i.e., MR-DoC needs a genetic instrument for X - constructed based on a GWAS of the exposure, and phenotypic measures for $\mathrm{X}$ and $\mathrm{Y}$ ). With MR-DoC and these rich phenotype resources - ranging from personality, diet and lifestyle to disease and psychiatric traits [see e.g.,(Hur and Craig 2013)] — collected at Twin and Family registries, the availability of genetic instruments robustly associated with the exposure remains the main limiting factor in addressing causal questions in non-experimental settings.

As presented here, MR-DoC is limited to twin data. Yet twin registries often have available information on additional family members (Hur and Craig 2013; Kaprio 2013; Skytthe et al. 2012; van Beijsterveldt et al. 2013; Willemsen et al. 2013). It is important to note that the method developed here can be generalized to sib data and within family tests. Additionally, as not all cohort studies necessarily include related individuals, we aim to expand the model, to accommodate distantly related individuals by using Genetic Relationship Matrices (GRMs) based on average allelic correlations (where the alleles are defined at the measured single nucleotide polymorphisms). We anticipate that these extensions will further increase statistical power and robustness to assumption violation (Keller et al. 2010; Posthuma and Boomsma 2000). Second, throughout the paper we assumed that the mating is random, there is no genotype-environment correlation, and no genotype by environment interaction. Indeed, assumption violation may also arise because the mating is assortative (Burgess and Thompson 2015), or because there are other plausible paths from the instrument to the outcome (except direct paths, or indirect, via the exposure), for example, via confounders affecting both traits, i.e., implying genotype by environment interaction or genotype by environment correlation. However, we note these effects may be captured by the MR-DoC twin model with appropriate experimental designs (Keller et al. 2010;
Plomin et al. 2013; Posthuma et al. 2003). On a cautionary note, although valid strong instruments are desirable in MR from the perspective of power, making up the polygenic score based on variants of unknown function should limit the testable hypotheses to whether the model is consistent or not with a direct causal effect from the exposure to the outcome [as pointed out by Burgess et al. (2014)]. While we considered the use of MR-DoC with polygenic scores, our conclusions also hold in scenarios where a genetic variant with known function is used as the instrument, which would improve the biological interpretation of the causal effect.

In conclusion, by integrating Mendelian Randomization with the Direction of Causation twin model, we developed a model that allows one to test and relax the strong 'no pleiotropy' assumption employed by standard MR. This approach therefore allows one to employ strong instrumental variables in the form of polygenic scores, guarding against weak instrument bias, and increasing the power to detect causal effects of exposures on potential outcomes.

We believe causal modeling in random samples (i.e., without experimental control) to be a considerable challenge, and subject to great interest and many developments [see e.g., (Burgess et al. 2017)]. It is unlikely-in the foreseeable future - that any single method or approach will be definite; it is more likely that the robust demonstration of causality will require evidence from multiple models and approaches (each with its own weaknesses and strengths). We believe that the approach presented here will make a valuable contribution to this undertaking. We anticipate that MR-DoC will enhance and extend MR's range of applications, and increase the value of the large cohorts collected at twin registries as they correctly detect causation and estimate effect sizes even in the presence of pleiotropy.

Acknowledgements This work was supported by the National Institute on Drug Abuse [Grant Number DA-018673].

\section{Compliance with ethical standards}

Conflict of interest Author C.C. Minica declares that she has no conflict of interest. Author C.V. Dolan declares that he has no conflict of interest. Author D.I. Boomsma declares that she has no conflict of interest. Author M.C. Neale declares that he has no conflict of interest. Author E. de Geus declares that he has no conflict of interest.

Informed Consent This article does not contain any studies with human participants performed by any of the authors.

Research involving human and animal participants This article does not contain any studies with human participants performed by any of the authors.

Open Access This article is distributed under the terms of the Creative Commons Attribution 4.0 International License (http://creativeco mmons.org/licenses/by/4.0/), which permits unrestricted use, distribution, and reproduction in any medium, provided you give appropriate 
credit to the original author(s) and the source, provide a link to the Creative Commons license, and indicate if changes were made.

\section{References}

Bekker PA, Merckens A, Wansbeek TJ (2014) Identification, equivalent models, and computer algebra: statistical modeling and decision science. Academic Press, Cambridge

Bollen KA, Bauldry S (2010) Model identification and computer algebra. Sociol Methods Res 39(2):127-156

Bound J, Jaeger DA, Baker RM (1995) Problems with instrumental variables estimation when the correlation between the instruments and the endogenous explanatory variable is weak. J Am Stat Assoc 90(430):443-450

Bowden J, Jackson C (2015) On the physical interpretation of a metaanalysis in the presence of heterogeneity and bias: from clinical trials to Mendelian randomization. arXiv arXiv: 150803768

Bowden J, Smith GD, Burgess S (2015) Mendelian randomization with invalid instruments: effect estimation and bias detection through Egger regression. Int J Epidemiol 44(2):512-525

Bowden J, Davey Smith G, Haycock PC, Burgess S (2016) Consistent estimation in Mendelian randomization with some invalid instruments using a weighted median estimator. Genet Epidemiol 40(4):304-314

Brion M-JA, Shakhbazov K, Visscher PM (2013) Calculating statistical power in Mendelian randomization studies. Int J Epidemiol 42(5):1497-1501

Bulik-Sullivan B, Finucane HK, Anttila V, Gusev A, Day FR, Loh P-R, Duncan L, Perry JR, Patterson N, Robinson EB (2015) An atlas of genetic correlations across human diseases and traits. Nat Genet 47(11): 1236

Burgess S, Thompson SG (2013) Use of allele scores as instrumental variables for Mendelian randomization. Int J Epidemiol 42(4):1134-1144

Burgess S, Thompson SG (2015) Mendelian randomization: methods for using genetic variants in causal estimation. CRC Press, Boca Raton

Burgess S, Thompson SG, Collaboration CCG (2011) Avoiding bias from weak instruments in Mendelian randomization studies. Int J Epidemiol 40(3):755-764

Burgess S, Freitag DF, Khan H, Gorman DN, Thompson SG (2014) Using multivariable Mendelian randomization to disentangle the causal effects of lipid fractions. PLoS ONE 9(10):e108891

Burgess S, Small DS, Thompson SG (2015) A review of instrumental variable estimators for Mendelian randomization. Stat Methods Med Res 26(5):2333-2355

Burgess S, Bowden J, Fall T, Ingelsson E, Thompson SG (2017) Sensitivity analyses for robust causal inference from mendelian randomization analyses with multiple genetic variants. Epidemiology 28(1):30-42

Davey Smith G, Ebrahim S (2003) 'Mendelian randomization': can genetic epidemiology contribute to understanding environmental determinants of disease? Int J Epidemiol 32(1):1-22

Davies NM, Hinke Kessler Scholder S, Farbmacher H, Burgess S, Windmeijer F, Smith GD (2015) The many weak instruments problem and Mendelian randomization. Stat Med 34(3):454-468

de Geus EJ (2006) Genetic pleiotropy in depression and coronary artery disease. Psychosom Med 68(2):185-186

De Moor MH, Boomsma DI, Stubbe JH, Willemsen G, de Geus EJ (2008) Testing causality in the association between regular exercise and symptoms of anxiety and depression. Arch Gen Psychiatry 65(8):897-905
Derks EM, Dolan CV, Boomsma DI (2006) A test of the equal environment assumption (EEA) in multivariate twin studies. Twin Res Hum Genet 9(03):403-411

Duffy DL, Martin NG (1994) Inferring the direction of causation in cross-sectional twin data: theoretical and empirical considerations. Genet Epidemiol 11(6):483-502

Evans DM, Davey Smith G (2015) Mendelian randomization: new applications in the coming age of hypothesis-free causality. Annu Rev Genomics Hum Genet 16:327-1350

Evans DM, Visscher PM, Wray NR (2009) Harnessing the information contained within genome-wide association studies to improve individual prediction of complex disease risk. Hum Mol Genet 18(18):3525-3531

Evans DM, Brion MJA, Paternoster L, Kemp JP, McMahon G, Munafò M, Whitfield JB, Medland SE, Montgomery GW, Timpson NJ (2013) Mining the human phenome using allelic scores that index biological intermediates. PLoS Genet 9(10):e1003919

Ference BA, Yoo W, Alesh I, Mahajan N, Mirowska KK, Mewada A, Kahn J, Afonso L, Williams KA, Flack JM (2012) Effect of longterm exposure to lower low-density lipoprotein cholesterol beginning early in life on the risk of coronary heart disease: a Mendelian randomization analysis. J Am Coll Cardiol 60(25):2631-2639

Ference BA, Majeed F, Penumetcha R, Flack JM, Brook RD (2015) Effect of naturally random allocation to lower low-density lipoprotein cholesterol on the risk of coronary heart disease mediated by polymorphisms in NPC1L1, HMGCR, or both: a $2 \times$ 2 factorial Mendelian randomization study. J Am Coll Cardiol 65(15):1552-1561

Gillespie NA, Zhu G, Neale MC, Heath AC, Martin NG (2003) Direction of causation modeling between cross-sectional measures of parenting and psychological distress in female twins. Behav Genet 33(4):383-396

Heath AC, Kessler RC, Neale MC, Hewitt JK, Eaves LJ, Kendler KS (1993) Testing hypotheses about direction of causation using cross-sectional family data. Behav Genet 23(1):29-50

Holmes MV, Lange LA, Palmer T, Lanktree MB, North KE, Almoguera B, Buxbaum S, Chandrupatla HR, Elbers CC, Guo Y (2014a) Causal effects of body mass index on cardiometabolic traits and events: a Mendelian randomization analysis. Am J Hum Genet 94(2): 198-208

Holmes MV, Asselbergs FW, Palmer TM, Drenos F, Lanktree MB, Nelson CP, Dale CE, Padmanabhan S, Finan C, Swerdlow DI (2014b) Mendelian randomization of blood lipids for coronary heart disease. Eur Heart J 1(36):539-550

Holmes MV, Dale CE, Zuccolo L, Silverwood RJ, Guo Y, Ye Z, Prieto-Merino D, Dehghan A, Trompet S, Wong A (2014c) Association between alcohol and cardiovascular disease: Mendelian randomisation analysis based on individual participant data. Bmj 349:g4164

Hur Y-M, Craig JM (2013) Twin registries worldwide: an important resource for scientific research. Twin Res Hum Genet 16(1):1-12

Johnson T, Johnson MT (2012) Package 'gtx'

Kaprio J (2013) The finnish twin cohort study: an update. Twin Res Hum Genet 16(1):157-162

Keller MC, Medland SE, Duncan LE (2010) Are extended twin family designs worth the trouble? A comparison of the bias, precision, and accuracy of parameters estimated in four twin family models. Behav Genet 40(3):377-393

Kendler KS, Neale MC, Kessler RC, Heath AC, Eaves LJ (1992) Major depression and generalized anxiety disorder: same genes,(partly) different environments? Arch Gen Psychiatry 49(9):716-722

Kleiber C, Zeileis A (2008) Applied econometrics with R. Springer, New York 
Kohler H-P, Behrman JR, Schnittker J (2011) Social science methods for twins data: integrating causality, endowments, and heritability. Biodemography Soc Biol 57(1):88-141

Ligthart L, Boomsma DI (2012) Causes of comorbidity: pleiotropy or causality? Shared genetic and environmental influences on migraine and neuroticism. Twin Res Hum Genet 15(02):158-165

Ligthart L, Hottenga J-J, Lewis CM, Farmer AE, Craig IW, Breen G, Willemsen G, Vink JM, Middeldorp CM, Byrne EM (2014) Genetic risk score analysis indicates migraine with and without comorbid depression are genetically different disorders. Hum Genet 133(2):173-186

Mather K, Jinks JL (2012) Introduction to biometrical genetics. Springer, New York

Middeldorp C, Cath D, Van Dyck R, Boomsma D (2005) The comorbidity of anxiety and depression in the perspective of genetic epidemiology. A review of twin and family studies. Psychol Med 35(05):611-624

Morgan MB, Geddes KO, Heal KM, Labahn G, Vorkoetter SM, McCarron J, DeMarco P (2005) Maple 10 programming guide. Maplesoft, Waterloo

Neale MC, Kendler KS (1995) Models of comorbidity for multifactorial disorders. Am J Hum Genet 57(4):935

Neale MC, Hunter MD, Pritikin JN, Zahery M, Brick TR, Kirkpatrick RM, Estabrook R, Bates TC, Maes HH, Boker SM (2016) OpenMx 2.0: extended structural equation and statistical modeling. Psychometrika 81(2):535-549

Palmer TM, Lawlor DA, Harbord RM, Sheehan NA, Tobias JH, Timpson NJ, Smith GD, Sterne JA (2012) Using multiple genetic variants as instrumental variables for modifiable risk factors. Stat Methods Med Res 21(3):223-242

Pickrell JK, Berisa T, Liu JZ, Ségurel L, Tung JY, Hinds DA (2016) Detection and interpretation of shared genetic influences on 42 human traits. Nat Genet 48(7):709

Pierce BL, Ahsan H, VanderWeele TJ (2010) Power and instrument strength requirements for Mendelian randomization studies using multiple genetic variants. Int J Epidemiol 40(3):740-752

Plomin R, DeFries JC, Knopik VS, Neiderheiser J (2013) Behavioral genetics. Palgrave Macmillan, Basingstoke

Posthuma D, Boomsma DI (2000) A note on the statistical power in extended twin designs. Behav Genet 30(2):147-158

Posthuma D, Beem AL, De Geus EJ, Van Baal GCM, von Hjelmborg JB, Iachine I, Boomsma DI (2003) Theory and practice in quantitative genetics. Twin Res Hum Genet 6(5):361-376

Proitsi P, Lupton MK, Velayudhan L, Newhouse S, Fogh I, Tsolaki M, Daniilidou M, Pritchard M, Kloszewska I, Soininen H (2014) Genetic predisposition to increased blood cholesterol and triglyceride lipid levels and risk of Alzheimer disease: a Mendelian randomization analysis. PLoS Med 11(9):e1001713

Purcell SM, Wray NR, Stone JL, Visscher PM, O’Donovan MC, Sullivan PF, Sklar P, Ruderfer DM, McQuillin A, Morris DW (2009) Common polygenic variation contributes to risk of schizophrenia and bipolar disorder. Nature 460(7256):748-752

R-Core-Team (2015) R: a language and environment for statistical computing. R Foundation for Statistical Computing, Vienna

Sheehan NA, Didelez V, Burton PR, Tobin MD (2008) Mendelian randomisation and causal inference in observational epidemiology. PLoS Med 5(8):e177
Sivakumaran S, Agakov F, Theodoratou E, Prendergast JG, Zgaga L, Manolio T, Rudan I, McKeigue P, Wilson JF, Campbell H (2011) Abundant pleiotropy in human complex diseases and traits. Am J Hum Genet 89(5):607-618

Skytthe A, Christiansen L, Kyvik KO, Bødker FL, Hvidberg L, Petersen I, Nielsen MMF, Bingley P, Hjelmborg J, Tan Q, Holm NV, Vaupel JW, McGue M, Christensen K (2012) The danish twin registry: linking surveys, national registers, and biological information. Twin Res Hum Genet 16(1):104-111

Smith GD (2011) Use of genetic markers and gene-diet interactions for interrogating population-level causal influences of diet on health. Genes Nutr 6(1):27-43

Smith GD, Hemani G (2014) Mendelian randomization: genetic anchors for causal inference in epidemiological studies. Hum Mol Genet 23(R1):R89-R98

Solovieff N, Cotsapas C, Lee PH, Purcell SM, Smoller JW (2013) Pleiotropy in complex traits: challenges and strategies. Nat Rev Genet 14(7):483-495

Turkheimer E, Harden KP (2000) Testing quasi-causal hypotheses using multivariate twin data In: Reis HT, Judd CM (eds) Handbook of research methods in social and personality psychology. Cambridge University Press, Cambridge

van Kippersluis H, Rietveld CA (2017) Pleiotropy-robust Mendelian randomization. Int J Epidemiol 2017:dyx002

van Beijsterveldt CE, Groen-Blokhuis M, Hottenga JJ, Franić S, Hudziak JJ, Lamb D, Huppertz C, de Zeeuw E, Nivard M, Schutte N (2013) The young Netherlands Twin register (YNTR): longitudinal twin and family studies in over 70,000 children. Twin Res Hum Genet 16(01):252-267

Van Der Sluis S, Dolan CV, Neale MC, Posthuma D (2008) Power calculations using exact data simulation: a useful tool for genetic study designs. Behav Genet 38(2):202-211

Verhulst B, Estabrook R (2012) Using genetic information to test causal relationships in cross-sectional data. J Theoret Polit 24(3):328-344

Verhulst B, Eaves LJ, Hatemi PK (2012) Correlation not causation: the relationship between personality traits and political ideologies. Am J Polit Sci 56(1):34-51

Vimaleswaran KS, Berry DJ, Lu C, Tikkanen E, Pilz S, Hiraki LT, Cooper JD, Dastani Z, Li R, Houston DK (2013) Causal relationship between obesity and vitamin D status: bi-directional Mendelian randomization analysis of multiple cohorts. PLoS Med 10(2): e1001383

Visscher PM, Yang J (2016) A plethora of pleiotropy across complex traits. Nat Genet 48(7):707

Visscher PM, Brown MA, McCarthy MI, Yang J (2012) Five years of GWAS discovery. Am J Hum Genet 90(1):7-24

Welter D, MacArthur J, Morales J, Burdett T, Hall P, Junkins H, Klemm A, Flicek P, Manolio T, Hindorff L (2014) The NHGRI GWAS Catalog, a curated resource of SNP-trait associations. Nucleic Acids Res 42(D1):D1001

Willemsen G, Vink JM, Abdellaoui A, den Braber A, van Beek JH, Draisma HH, van Dongen J, van't Ent D, Geels LM, van Lien R (2013) The Adult Netherlands twin register: twenty-five years of survey and biological data collection. Twin Res Hum Genet 16(01):271-281 